Las dedicatorias en las novelas juveniles argentinas

Damián Nicolás Martínez

Revista Argentina de Estudios de Juventud, (14), e036, 2020

ISSN 1852-4907 | https://doi.org/10.24215/18524907e036

https://perio.unlp.edu.ar/ojs/index.php/revistadejuventud

FPyCS | Universidad Nacional de La Plata

La Plata | Buenos Aires | Argentina

\title{
LAS DEDICATORIAS \\ EN LAS NOVELAS JUVENILES ARGENTINAS
}

\section{Dedications in Argentinian Young Adult Novels}

\section{Damián Nicolás Martínez}

dnmartinez@campus.ungs.edu.ar

https://orcid.org/0000-0003-0295-1259

Instituto del Desarrollo Humano

Universidad Nacional de General Sarmiento

Argentina

\section{Resumen}

Palabras clave

dedicatorias literatura juvenil paratexto Argentina

\section{Keywords}

dedications youth literature paratext Argentina
El artículo explora las dedicatorias escritas por autores/as de novelas juveniles argentinas, publicadas en el período 1983-2020. En primer lugar, se abordan las dedicatorias en función de algunas categorías planteadas por Gerard Genette. Luego, se analizan en profundidad las dedicatorias de novelas de temas difíciles y las novelas de temática sobre inmigración. Por último, se reflexiona sobre la riqueza del abordaje de las dedicatorias junto con otros elementos paratextuales (prólogos, introducciones, etc.) para leer novelas juveniles.

\section{Abstract}

This article explores the dedications written by authors in Argentinian novels for young people, published in the period 1983-2020. In the first place, dedications are dealt with based on some categories proposed by Gerard Genette. Then, the dedications of hard topics novels and novels about inmigration are deeply analyzed. Finally, it reflects on the wealth of addressing a book regarding other paratexts (prologues, introductions, etc.) while reading young adults novels. 


\title{
LAS DEDICATORIAS \\ EN LAS NOVELAS JUVENILES ARGENTINAS
}

\author{
Por Damián Nicolás Martínez
}

«Pido perdón a los niños por haber dedicado este libro a una persona grande», escribió Antoine de Saint-Exupéry (1943) en la página anterior del relato que se convertiría en el libro infantil más vendido de la historia. «En homenaje de mi nostalgia a mi hermano Luis, el Rey Luis, y a mi hermana Gloria; Luis renunció a vivir a los veinte años, y Gloria a los veinticuatro también pensó que realmente vivir no valía la pena», expresó José Mauro de Vasconcelos (1968) al presentar la que sería la novela brasileña más leída por los/as jóvenes argentinos/as. «A la juventud de América», son las palabras con que José Enrique Rodó (1900) fusionó hace 120 años la dedicatoria y la explicitación del destinario previsto de su ensayo. «Este libro está dedicado a todos esos chicos que tienen más amigas que amigos, su voz suena más aguda de lo normal y caminan distinto. A los niños a los que llaman “maricón” por los pasillos del colegio. A quienes los defienden», redactó un adolescente español, conocido como Peter Pan en las redes sociales, cuando firmó como Christian Martínez Pueyo (2016) la novela que a los veinte años le daría fama internacional.

Estas sugestivas y, quizás, hasta recordadas dedicatorias pertenecen a los libros El principito (1943), Mi planta de naranja lima (1968), Ariel (1900) y El chico de las estrellas (2016), respectivamente. A diferencia de las mencionadas, muchas dedicatorias que los/as autores/as incluyen en sus obras son omitidas en su lectura, olvidadas, poco valoradas. Incluso, se las confunde con las frases que escribe quien regala un libro, o ni siquiera son fotocopiadas cuando una obra debe ser leída en las aulas de educación secundaria. Por el contario, desde nuestra perspectiva, consideramos que las dedicatorias de los libros destinados a jóvenes tienen valor. Entendemos que se trata de frases que, en pocas líneas (o incluso, en una sola), pueden poner en tensión las relaciones entre los aspectos autobiográficos y aquellos temáticos o argumentales del libro que preceden. 
Desde esta concepción, en el presente trabajo se abordan las dedicatorias que expresan los/as autores/as en las novelas juveniles publicadas en la Argentina. Consideramos, principalmente, aquellas obras incluidas en colecciones o en series destinadas a su lectura en el ámbito escolar. En menor medida, tenemos en cuenta la narrativa para jóvenes lectores/as en general. En cuanto al recorte temporal, nos abocamos a las novelas publicadas desde comienzos de la transición democrática, ya que es el momento en que las editoriales comienzan a incluir literatura juvenil en colecciones escolares. Este recorte abarca hasta la actualidad, por lo que se trata del período que comprende de 1983 al presente.

A partir de una exploración por las novelas juveniles argentinas, presentamos, en primer lugar, un escueto panorama por diferentes tipos de dedicatorias. Posteriormente, indagamos las posibles funciones de algunas dedicatorias en particular. De esta manera, aspiramos a generar conocimiento acerca de representaciones sobre el hecho literario y el espacio escolar propuestas por textos del canon juvenil de circulación en la escuela media.

\section{La dedicatoria en el marco de los paratextos}

Partimos de la noción de paratexto, entendido como «dispositivo pragmático que, por una parte, predispone -o condiciona- para la lectura y, por otra, acompaña en el trayecto, cooperando con el lector en su trabajo de construcción -o reconstrucción- del sentido» (Alvarado, 2006, p. 10). En este sentido, abordamos como paratexto «aquello por lo cual un texto se hace libro y se propone como tal a sus lectores, y, más generalmente, al público» (Genette, 2001, p. 13). A su vez, tomamos la distinción entre paratextos de autor, paratextos de editor y paratextos de terceros (p. 13). Con el fin de ajustar precisiones teóricas sobre nuestro objeto, recurrimos a la obra Umbrales (2001), de Gerard Genette, cuyo capítulo «Dedicatorias» presenta conceptualizaciones con las que abordamos nuestro análisis.

Quizás resulte una obviedad, pero como lo que resulta obvio no se expresa -y si se expresa es porque no resulta obvio-, aclaramos que para analizar las dedicatorias solo tomamos aquellas novelas juveniles argentinas que cuentan con ellas. 
Tal como plantea Genette (2001), «la ausencia de dedicatoria en un sistema que implica la posibilidad es significativa como un grado cero. "Este libro no está dedicado a nadie”: ese mensaje implícito ¿no está lleno de sentido?» (pp. 115-116). De acuerdo con esta perspectiva, podrían analizarse las novelas sin dedicatorias, pero tal estudio está fuera del marco del presente trabajo.

Genette (2001) distingue entre «dedicatoria de obra y dedicatoria de ejemplar» (p. 117). Considera que «las dos consisten en hacer el homenaje de una obra a una persona, a un grupo real o ideal». No obstante, mientras que la primera se vincula con «la realidad ideal de la obra misma, cuya posesión [...] no puede ser más que simbólica»; la segunda «implica la realidad material de aquel ejemplar que se dona o se vende efectivamente» (p. 101). Para el presente trabajo, abordamos solo la primera categoría. Es decir, las dedicatorias que los/as autores/as hacen imprimir en la edición de sus libros ${ }^{1}$ y que consisten en

[...] la exposición (sincera o no) de una relación (de una clase o de otra) entre el autor y alguna persona, grupo o entidad [que, a su vez] destaca siempre la demostración, la ostentación, la exhibición: exhibe una relación intelectual o privada, real o simbólica, y esta exhibición está siempre al servicio de la obra como argumento de valorización o tema de comentario (Genette, 2001, p. 116).

En cuanto a la ubicación en el libro, su «emplazamiento canónico [...] desde finales de siglo XVı es evidentemente el principio del libro y, más precisamente hoy, sobre la primera página después de la portadilla» ${ }^{2}$ (p. 109).

En las dedicatorias, la acción de dedicar se cumple con la mención de la persona, grupo o institución a la que se dedica la obra, es decir, «su función propia se agota en esta exposición»(Genette, 2001, p. 114). Por lo tanto, se trata de una situación performativa, ya que lo que se dice se constituye en acto. Es más, lo que está implícito también, ya que es más habitual que comiencen con «a...» o «para...», en vez de «dedico este libro a...». De todas maneras, por tratarse de una obra de carácter público, contamos con dos tipos de destinatarios: 
Sea quien fuere el dedicatario oficial, hay siempre una ambigüedad en la destinación de una dedicatoria de obra, que apunta siempre al menos a dos destinatarios: el dedicatario, por supuesto, pero también el lector, ya que se trata de un acto público en el que el lector es de alguna manera tomado como testigo. Típicamente performativa [...], ya que constituye el acto que está describiendo (Genette, 2001, p. 109).

Sin embargo, se registran «usurpaciones adicionales sobre las del prefacio» (Genette, 2001, p. 114). Dado esto, también nos interesa analizar en qué tipo de novelas juveniles aparecen estas dedicatorias. De hecho, el presente trabajo hace foco en la relación que las dedicatorias establecen con otros elementos paratextuales y, sobre todo, con otros aspectos del libro. Por ello, recurrimos a la pauta teórica que Gemma Lluch (2004) desarrolla en Cómo analizamos relatos infantiles y juveniles. La autora propone abordar los libros de literatura infantil y juvenil a través de un análisis que comprende tres fases. La primera consiste en considerar el momento y los responsables de la creación y de la recepción del libro. La segunda, hace hincapié en aquellos elementos paratextuales que aparecen en el libro, pero que todavía no son la historia. La tercera, se concentra en el relato (Lluch, 2004). Este marco nos habilita a analizar las dedicatorias en relación con aquellos elementos involucrados en las otras fases. Es decir, indagamos cómo las dedicatorias que los/as autores/as expresan en sus libros se vinculan tanto con las condiciones de creación y de recepción de las obras (comprendidas en la primera fase del análisis) como con los textos literarios (objeto de la tercera fase).

En su análisis sobre la literatura juvenil, plasmado en el artículo «"Pero a lxs chicxs les gusta” y otros cortocircuitos en la literatura juvenil» (2019), Paula Labeur omite referirse a las dedicatorias. Sin embargo, cuando brinda como propuesta didáctica para las clases de Literatura la actividad de redactar prólogos para las obras de literatura juvenil, aclara que esta tarea «puede llevar a discutir largamente para qué y quién escribe los prólogos, y para qué están y cómo se constituyen esas dos tribus lectoras de lxs que leen y lxs que no leen los prólogos» (Labeur, 2019, p. 71). Consideramos que es posible ampliar su abordaje sobre los prólogos hacia los otros elementos paratextuales que pueden encontrarse en las novelas juveniles; en nuestro caso, las dedicatorias, lo que nos permite enmarcarnos en la «tribu lectora» de los/as que leen tanto los prólogos como las dedicatorias. 


\section{Las dedicatorias en las novelas juveniles: posibles categorías}

A la noción general de dedicatoria, entendida como «la exposición [...] de una relación [...] entre el autor y alguna persona, grupo o entidad» (Genette, 2001, p. 116), sumamos la de dedicatario privado, entendido como «una persona conocida o no por el público a quien es dedicada una obra en nombre de una relación personal: de amistad, familiar u otra» (p. 113), como así como el rasgo que Lluch (2004) plantea en su propuesta sobre el análisis de los paratextos en la literatura infantil y juvenil: «Las dedicatorias mayoritariamente destacan una relación afectiva entre el autor y la persona a quien va dirigida, que pertenece habitualmente al círculo familiar» (p. 55).

Esta clase de dedicatoria, a la que podríamos denominar afectiva, es una de las más habituales en las novelas juveniles. Hijos/as, padres, parejas, hermanos/as o personas que se nombran sin explicitar el vínculo son mencionados/as, a veces, con algún detalle que da cuenta de la afinidad entre autores/as y dedicatarios/as. A modo de ejemplo, podemos presentar las siguientes: ${ }^{3}$

A Josefina.

María Teresa Andruetto (2016 [2005]). Veladuras.

Para mi mamá.

Marcelo Birmajer (1993). Derrotado por un muerto.

A mi padre.

Liliana Bodoc (2000). Los días del venado.

Para Silvia, la que llegó un día de la mano de Hugo y trajo su genuino silencio a una mesa demasiado estruendosa... Para ella, para honrar su nuevo nacimiento. Liliana Bodoc (2013). El perro del peregrino.

Para Luciano, por haberme enseñado que hay muchas maneras de estar cerca. Ezequiel Dellutri (2018). Koi.

A mi hijo Gabriel por todo lo que de él aprendo cada día.

Alma Maritano (1984). El visitante. 
A Jimena Inés, por su claridad, por su alegría.

Alma Maritano (1986). Vaqueros y trenzas.

A Miguel Ángel.

Alma Maritano (1997). Pretextos para un crimen.

Para Sandra, por supuesto.

Antonio Santa Ana (1998). Los ojos del perro siberiano.

Para Cecilia, que me hizo llegar la capa justo a tiempo.

Juan Solá (2019). Naranjo en flúo.

En estos casos, todas las dedicatorias tienen un/a único/a destinatario/a. No obstante, en las novelas juveniles se registran ejemplos de una clase de dedicatoria a la que Elsa Bornemann (1994) denomina dedicatoria colectiva. Se trata, tal como esta autora lo ejemplifica en Socorro (1994), su antología de cuentos de terror, de dedicatorias en las que se mencionan varias personas. Algunos ejemplos serían los siguientes:

Para Dany y Edu, con quienes, cuando llegaba el verano, por la cantidad de materias que nos habíamos llevado, decíamos: «Bueno, empezaron las clases». Marcelo Birmajer (1992). Un crimen secundario.

Para Felipe y Martín, dos niños luminosos que, a veces, son artistas del color y la forma. A veces, ángeles. A veces, monjes rubios de un templo chino. Para Patricia y el «Tucu», sus padres.

Liliana Bodoc (2013). El espejo africano.

A mis hermanas, Silvina y María Laura.

Pablo De Santis (2003). El inventor de juegos.

Para Alejo, Rodrigo y Violeta.

Norma Huidobro (2004). Octubre, un crimen.

A Olga, siempre al pie del cañón. A nuestras familias, que (un poco) nos hicieron como somos. A todas nosotras.

Patricia Kolesnicov (2017). Me enamoré de una vegetariana.

A Mateo, Federico, Florencia y Héctor porque los elijo cada día. Margarita Maine (2005). El hijo de la libertad. 
A Lucía, Tomás y Ramiro.

Claudia Piñeiro (2010). El fantasma de las invasiones inglesas.

A Nuncio, Julio, Fede, Martina, Juan Pablo, Homerito, Felipe, Camilo y Paloma.

Pablo Ramos (2015). El sueño de los murciélagos.

Para mis padres, para mis hijos.

Verónica Sukaczer (2015). Los nombres prestados.

En algunos casos, la lectura de las dedicatorias en orden cronológico puede brindar algún tipo de indicio sobre aspectos biográficos de quien escribe en relación con sus dedicatarios. En efecto, a partir de las dedicatorias que se reproducen a continuación puede rastrearse la relación entre la autora y alguien apodado Lolo. En la primera, se menciona en tiempo presente un vínculo estrecho entre Paula Bombara y Lolo, mientras que en la tercera, escrita años después, se lo menciona en pasado, pero se destaca que algo (o alguien) los une en el presente:

Dedico esta novela a mis padres, por ser Poesía y por ser Coraje.

$Y$ a Lolo, por abrazarme cuando el miedo juega con mi espalda.

Paula Bombara (2005 [2002]). El mar y la serpiente.

A mamá. Por los libros, por la música, por el arte.

Por los cielos abiertos, por los abrazos.

Paula Bombara (2015). La chica pájaro.

A Lolo. Por lo que nos unió. Y también por lo que nos une.

Paula Bombara (2016). Lo que guarda un caracol.

Examinamos, a continuación, dos dedicatorias de la escritora Andrea Ferrari:

Para Ernesto y Valeria.

Andrea Ferrari (2003). El complot de las flores.

Para Ernesto y Valeria por todo.

Andrea Ferrari (2014 [2007]). El camino de Sherlock.

Esta lectura parcial de la bibliografía de la autora puede llevar a pensar que todas sus novelas se dedican a las mismas personas. De esa manera, se enmarcarían en alguna clase de dedicatoria que evocaría a Abelardo Castillo, quien expresó en 
todos sus libros de relatos una dedicatoria a la misma persona y así lo plasmó en Cuentos completos (1997): «Todos mis cuentos los ya escritos y los que aún quedan por escribir pertenecen a un solo libro incesante y a una mujer, a Sylvia, quien le dio a ese libro el nombre que hoy lleva: Los mundos reales». Es decir, una dedicatoria a lo Castillo sería aquella perteneciente a un conjunto de dedicatorias que en todos los libros evoca a la/s misma/s persona/s.

Un recorrido por otras novelas de Ferrari confirma que sus obras no se ajustan a esta categoría. Es más, este tipo de dedicatorias no se registran en las novelas juveniles argentinas. ${ }^{4}$ Así lo demuestran los siguientes casos:

A mi madre que me contagió el placer por los libros.

Andrea Ferrari (2004). Café solo.

A Inés, que me ayuda a evitar que se rebelen las palabras.

Andrea Ferrari (2015 [2004]). La rebelión de las palabras.

\section{Del afecto a la novela}

En una primera exploración por las novelas juveniles, encontramos algunas dedicatorias que no se ajustan exclusivamente a las caracterizaciones desarrolladas. Esto nos lleva a pensar en otras categorías posibles. Para ello, consideramos qué expresan aquellas dedicatorias que no involucran solo la dimensión afectiva. Es decir, aquellas en las cuales los/as autores/as hacen algo más que mencionar a las personas que aprecian.

En este sentido, identificamos un grupo numeroso de dedicatorias que explicitan una vinculación con el contenido del libro que preceden. Es decir, personas a las que se les dedica un libro no solo por afinidad sentimental sino también por algún tipo de relación temática o argumental que mantienen con la obra. Se trata, en estos casos, de una «dedicatoria motivada, en donde la motivación toma generalmente la forma de una breve caracterización del dedicatario y/o de la forma dedicada» (Genette, 2001, p. 108). Este tipo de dedicatorias motivadas puede ejemplificarse con los siguientes casos: 
Yo no sé vivir sin el rumor de los otros a mí alrededor. Jamás supe estar sola.

Este libro, que cuenta encuentros y alianzas, es para los que sostienen el rumor de sus vidas: Odino, que me rescató de la desesperanza, y nuestros tres hijos,

Dante, Tamara, Selva, que cuando dicen mi nombre, me hacen de nuevo, desde afuera.

Márgara Áverbach (2009). Una cuadra.

A la gente del Comedor, por haberme invitado a su mesa.

A mis hijas, por su apoyo. A Franco.

María Laura Dedé (2015). El comedor de las tinieblas.

A Sara Mondani, que siempre supo ovillar historias.

Graciela Montes (1994). Otroso.

A mis amigos atletas, Víctor y María.

Marisa Potes (2018). El campo deportivo.

Para mi hija Maya. Y para Leo- ipor supuesto!

Monika Schillat (2013). Cartas a Messi.

Si bien los títulos brindan algún indicio sobre las motivaciones que evidentemente llevaron a estas autoras a designar tales dedicatarios, la lectura completa de las novelas o, por lo menos, un acercamiento a sus argumentos, complementa el sentido.

En Otroso (1994), se retoman elementos de la mitología griega para instalarlos en un nuevo escenario: la localidad de Florida, en el Gran Buenos Aires. Allí, en un mundo subterráneo llamado Otroso, Ariadna, Tere, Hugo y varios amigos más viven enredadas aventuras que cuenta su narrador protagonista. Estas historias se vinculan en varios aspectos con la del hilo que Ariadna dio a Teseo cuando entró en el laberinto del Minotauro. De este tejido da cuenta la autora cuando plantea que la dedicataria «siempre supo ovillar historias». Una cuadra (2009) cuenta el proyecto de un grupo de vecinos para embellecer los frentes de las casas de toda la cuadra, personas que «sostienen el rumor de sus vidas», tal como la autora expresa. Cartas a Messi (2013), en tanto, se forma a partir de las cartas que los tripulantes de un buque de expedición le escriben a Leonel Messi para proponerle llevarlo de viaje a la Antártida para su cumpleaños. De allí que este jugador de fútbol sea tanto el destinatario de las cartas de la historia como uno de los dedicatarios del libro. 
El comedor de las tinieblas (2015) narra la historia de Washington, un joven uruguayo que se encuentra en España de visita en casa de un amigo. Como no quiere volver a Montevideo para iniciar sus estudios universitarios, empieza a trabajar en un restaurante temático cuyo nombre le da título a la novela. De ese ámbito, un comedor, se toma la autora para desarrollar su dedicatoria. En El campo deportivo (2018), dos escuelas históricamente enfrentadas deben resolver el problema que se le presenta a una de ellas que está a punto de perder el lugar que le da título a la novela. Su autora explicita la actividad que ejercen sus dedicatarios.

Las dedicatorias descriptas tienen dedicatarios que pueden ser individualizados. En cambio, se registran otras que también dan cuenta del afecto, la motivación y lo colectivo, pero que presentan un matiz particular. Se trata de aquellas destinadas a «un grupo» (Genette, 2001, p. 114) con características homogéneas, a un destinatario colectivo cuyos miembros tienen en común mucho más que ser mencionados por un/a autor/a en una misma página. Un ejemplo de esta categoría es la de Franco Vaccarini (2015) en Cazadores: "A mi amigo Eduardo Petralía. A los cazadores arrepentidos». También puede incluirse en este grupo la particular dedicatoria de Las botas de Anselmo Soria (1998), de Pedro Orgambide: «Cuando era muchacho, me hubiera gustado leer una historia como esta. Por eso, la escribo para vos». ${ }^{5}$ Aunque redactada en singular, apela a un grupo con el que el propio autor se siente identificado.

\section{Las dedicatorias en las novelas de temas difíciles}

Dentro de la categoría de dedicatorias motivadas, se observan algunas que incluyen ciertas menciones que buscan dejar en claro que el libro escrito es una especie de revancha. ${ }^{6}$ En general, aparecen en obras que Graciela Perriconi (2005) denomina literatura de temas difíciles en el marco de la literatura infantil y juvenil:

Los temas difíciles, por dar un nombre de referencia, son aquellos que están relacionados con las situaciones límites, las que nos enfrentan con nuestra mismidad, sin concesiones, que nos motivan necesariamente a preguntarnos por el sentido de la vida y por el sentido de la muerte, por las ausencias y la enfermedad, en síntesis, aquellas situaciones que nos enfrentan al casi inagotable interrogante del para qué y por qué vivimos (Perriconi, 2005, p. 64). 
Entre las novelas juveniles de temas difíciles predominan las dedicatorias con múltiples destinatarios/as. Así, en muchas de ellas se apela a grupos de carácter público como las agrupaciones Madres y Abuelas de Plaza de Mayo, en novelas que abordan la temática de los desaparecidos durante la última Dictadura cívico militar argentina; a grupos que podríamos denominar anónimos pero que son homogéneos como niños bajo tratamientos oncológicos en una novela que aborda el cáncer; a entes abstractos como la verdad, la memoria y la dignidad junto con personas a las que se menciona por su nombre de pila o su nombre y apellido e, incluso, profesión y motivación.

Los temas difíciles de estas novelas podrían resumirse de la siguiente manera: la recuperación de la identidad de jóvenes hijos/as de personas desaparecidas, en El año de la vaca (2003), Cruzar la noche (1995) y Los sapos de la memoria (1997); el abuso sexual, en El infierno de los vivos (2015) y la lucha contra el cáncer en Si tu signo no es cáncer (2004). Se expone, a continuación, este panorama de dedicatorias:

A las abuelas de Plaza de Mayo, que conocen la historia. A Mónica, María Cristina, Diana Perla, Miriam, Lea, que se sentaron conmigo a conversar en bares, en patios, en bancos de plaza hasta que me devolvieron, de a poco, con paciencia infinita, la conciencia del poder que hay en la charla. Márgara Áverbach (2003). El año de la vaca.

A las Madres y Abuelas de Plaza de Mayo. A todas las víctimas del terrorismo de Estado. A la verdad y a la memoria.

Alicia Barberis (1995). Cruzar la noche.

A Elba V., protagonista de esta historia. Donde quiera que esté.

A la dignidad de las mujeres.

Alicia Barberis (2015). El infierno de los vivos.

A Mario y su rebelde pasión por la libertad. A Líbele Cinman y su familia, que enseñaron la solidaridad del abrazo oportuno. A mis compañeros de la Escuela de Ciencias de la Información, de la Universidad Nacional de Córdoba, arrancados, desaparecidos, torturados y asesinados por la dictadura militar implantada en Argentina en 1976. A mi querido amigo Hugo Kogan, a quien le extirparon para siempre las primaveras. A mis «sobrinos» Margarita y Daniel Caffaratti, a quienes les amputaron un papá y aún siguen caminando su destino. 
A las madres, abuelas e hijos que todavía buscan justicia. A Mirella, un oasis de amistad en medio de tanta muerte. $Y$ a todos los que sobrevivimos con la estúpida culpa de estar vivos.

Graciela Bialet (1997). Los sapos de la memoria.

A Julián y Magdalena por resistir. A Leticia y Florencia por estar en el momento justo. A Silvio Marchegiani y Ricardo Chiosso, médicos que atienden a las personas y no solo a sus enfermedades. A los superniños calvos... iQué sería de las salas oncológicas sin sus proezas! A la entrañable Graciela Cabal que me impulsó a escribir esta historia.

Graciela Bialet (2004). Si tu signo no es cáncer.

Algunas de las dedicatorias de este grupo establecen relación con otros elementos paratextuales de las obras a las que pertenecen. De hecho, la dedicatoria «A mi hermana, para que no muera su nombre», de Mientras no muera tu nombre (2019), de Liliana Cinetto, es retomada al final de la novela, donde la autora menciona explícitamente su vinculación personal con la temática de la violencia de género y el destino fatal de su hermana, la dedicataria. ${ }^{7}$ A este grupo podría pertenecer, también, la llamativa dedicatoria de Yael y la casa violeta (2018), de Julia Inés Mamone y María Ibarra. En este caso, autora e ilustradora se dedican el libro mutuamente, lo que permite considerar esta situación como una variante de «autodedicatoria o dedicatoria al autor por él mismo» (Genette, 2001, p. 115).

A su vez, estas autoras y dedicatarias se enmarcan como pertenecientes a un conjunto que podríamos denominar sobrevivientes: «A María por escribir, a Julia por dibujar y a las dos por sobrevivir». Este libro, sin textos en la contratapa, en el lomo ni en las solapas, fue escrito por Ibarra e ilustrado por Mamone y aborda la temática trans en la niñez, a partir de la historia de Yael quien es llevada por su mamá a la casa violeta donde conoce personas con quienes comparte inquietudes sobre su identidad de género.

\section{Dedicatorias a escritores/as}

Se observan dedicatorias en las que el/la autor/a menciona a otro/a escritor/a; es decir, dedicatorias que cuentan con un dedicatario público, entendido como «una persona más o menos conocida con quien el autor manifiesta tener, por su 
dedicatoria, una relación de orden público: intelectual, artístico, político u otros» (Genette, 2001, p. 114). En su realización, esta clase presenta dos variantes. Por un lado, una simple cuestión de afinidad, de amistad o de compañerismo declarado por lo que se entrelazan lo público y lo privado. Tal es el caso de la dedicatoria de Pablo De Santis (2008), en El buscador de finales, "A Marcelo Birmajer»; y la de Eduardo Muslip (1997), en Hojas de la noche, "A Hebe Uhart. A mis padres, a mi hermana». Por último, citamos la dedicatoria ya mencionada de Bialet (2004) en Si tu signo no es cáncer, libro dedicado, entre otras personas, «a la entrañable Graciela Cabal».

Por otro lado, se observa una variante en la que el/la autor/a menciona a un/a escritor/a a quien ubica como referente o como predecesor. Para su análisis, nos enfocamos en tres aportes al respecto. En primer lugar, consideramos a Jorge Luis Borges (1989 [1952]), quien identifica un procedimiento similar en su ensayo «Kafka y sus precursores» cuando afirma: «Cada escritor crea sus precursores. Su labor modifica nuestra concepción del pasado, como ha de modificar el futuro». A continuación, incorporamos a Harold Bloom (1994) quien llega a la noción de canon a partir del hecho de que algunos/as escritores/as sean retomados/as por otros/as autores/as. Al respecto, afirma que «se ha convertido en una elección entre textos que compiten para sobrevivir" y especifica que esta elección es realizada por «autores de aparición posterior que se sienten elegidos por figuras anteriores concretas» (Bloom, 1994, p. 30).

Por último, destacamos la dedicatoria in memoriam. Según Genette (2001), «la dedicatoria a título póstumo permite también exhibir una filiación intelectual» (p. 113). El caso más notable de esta variante sería la dedicatoria de Graffiti ninja (2007), escrita por Osvaldo Aguirre y por Eduardo González. ${ }^{8}$ Esta novela está precedida por la siguiente frase: «A Héctor Germán Oesterheld y Francisco Solano López, eternautas de la aventura». Oesterheld (desaparecido en 1977 por la última Dictadura cívico militar argentina) y Solano López son el escritor y el dibujante, respectivamente, de El eternauta (1957-1959), por lo que esta dedicatoria concentra varios aspectos. La intertextualidad se genera con la referencia a la emblemática historieta, a la que se recurre también a lo largo de la novela. El narrador protagonista, un periodista novato que comienza a trabajar en un diario sensacionalista, investiga una serie de crímenes y descubre junto a los cadáveres grafittis con citas de El eternauta. A su vez, esta historieta funciona para exponer la representación del narrador sobre la literatura: 
Una vez más recordé la imagen de El eternauta y, por un momento, creí estar alucinando. Comprendí que teníamos algo en común con los personajes de la historieta. Algo importante. No había ningún héroe, nadie tenía superpoderes. Nosotros, como ellos, éramos personas comunes que de pronto, sin quererlo, se encontraban en medio de una aventura. Una aventura que nos elegía como sus personajes. Y había que hacer con lo que sabíamos, como podíamos. Los trucos y las soluciones mágicas existían solamente en la ficción. $\mathrm{O}$ en las malas ficciones, mejor dicho (González \& Aguirre, 2007, pp. 134-135).

En síntesis, Graffiti ninja (2007) presenta la particularidad de haber sido escrita en coautoría y de que sus autores se la dedican a quienes, justamente, también escribieron bajo esta modalidad. Esta dedicatoria, in memoriam a medias, puede considerarse como un procedimiento que consistiría en nombrar precursores, una especie de intento de situarse junto con los personajes ya canónicos, en una filiación que los autores arman y en la que se inscriben.

Entre esta clase de dedicatorias, resulta importante destacar una que reúne ambas variantes. Se trata de la incluida por Carolina Unrein (2019) en Pendeja. Diario de una adolescente trans: «A Björk por enseñarme otros mundos y misiones posibles, a Carolina por abrirme la puerta de entrada, a Susy y a Camila por hacernos carne, a Papá y a Mamá por permitirme vivirlos». Tal como su subtítulo lo indica, no constituye específicamente una novela, sino un diario en el que se incluyen relatos sobre situaciones en torno del reconocimiento de la identidad de género de su autora, poemas que abordan su situación amorosa y algunas fotografías que ilustran su vida familiar. En la dedicatoria se identifica el componente afectivo tanto por la mención de sus padres como de otras cuatro personas. En primer lugar, le dedica el libro a la cantante, actriz y escritora islandesa Björk Guðmundsdóttir, conocida por su nombre de pila. A partir de esa referencia sin apellido, tampoco menciona los apellidos de las otras personas que nombra: alguien que se llama como ella, Susy y Camila.

Es necesario recurrir, una vez más, a la metodología que Lluch (2004) propone para concentrarnos en la primera fase del análisis de la literatura infantil y juvenil: el momento y los responsables de la creación y la recepción del libro. De este modo, si se tienen en cuenta elementos biográficos de Unrein y del contexto de producción de su obra, puede intuirse a quiénes se refiere en su dedicatoria. Podría 
tratarse de las actrices y escritoras Susy Shock y Camila Sosa Villada, ambas personas trans que cuentan con varias publicaciones que abordan aspectos autobiográficos. Shock, quien se reconoce como artista trans sudaca, publicó las antologías de poemas Revuelo sur (2007), Poemario Trans Pirado (2011) y Crianzas (2016), este último, con ilustraciones de Anahí Bazán Jara, reúne mensajes y reflexiones sobre la infancia. Sosa Villada, quien se identifica como travesti, lleva publicados La novia de Sandro (2015), libro que reúne los poemas que publicaba en su blog, El viaje inútil. Trans/escritura (2018), donde entrelaza episodios autobiográficos con reflexiones sobre su escritura, y Las malas (2019), novela que narra y describe la noche travesti cordobesa. Además, es autora del prólogo de Fatal. Una crónica trans (2020), el libro posterior de Unrein. Por lo expuesto, podemos considerar que la dedicatoria de esta autora resulta un procedimiento que consistiría en nombrar antecesoras pero relativitizado por dos cuestiones: por la omisión de los apellidos y por la fuerza del componente afectivo, ya que las invocadas son personas con quienes tendría un trato frecuente.

\section{Las dedicatorias en novelas sobre la inmigración}

En su artículo «La inmigración en la literatura juvenil», Marco Kunz (2003) identifica en la literatura española «el hecho de que los inmigrantes niños o adolescentes sólo desempeñan un papel importante en textos que se dirigen a un público que tiene más o menos la misma edad que los personajes» (pp. 38-39). Como si ese planteo también fuera válido a modo de panorama de la literatura argentina del siglo XXI, los libros con protagonistas inmigrantes que llegan a la Argentina entre finales del siglo XIX y principios del siglo XX conforman una especie en la novela juvenil local. Un ejemplo simple de estas dedicatorias se encuentra en El juramento de los Centenera (2007), de Lydia Carreras de Sosa: «A Edu, mi esposo hace 37 años». Incluso, podría incluirse en la primera categoría debido a su exclusivo componente afectivo.

Sin embargo, en las novelas juveniles sobre la inmigración se observan dedicatorias que, con rasgos similares a los de las categorías ya abordadas, presentan otras cualidades. En este grupo, Adriana Vulponi (2010) identifica que «aparece en las dedicatorias y en las voces de escritores [...] la memoria que atraviesa las 
generaciones, de abuelos y padres a hijos» (p. 268). Según esta autora, «otro "lugar" es rescatado allí, la "escucha de la voz antigua de los padres": contar la memoria es lo posible, lo necesario» (p. 268).

En primer lugar, se hacen menciones a inmigrantes, de quienes se tomaron algunos aspectos biográficos para escribir las novelas. Por lo tanto, se trata de una «dedicatoria al héroe, es decir a su principal objeto»(Genette, 2001, p. 115). Este sería el caso de El pan de la serpiente. Lazos de amistad durante la inmigración (2010), de Norma Huidobro, que está dedicada «a la memoria de Felisa Santa María y de Juana Echarri, mis abuelas». En el marco de los abusos padecidos de parte de personajes de la clase alta argentina que rechaza y se aprovecha de la masa de inmigrantes, la novela narra la historia de dos amigas, Felisa, «la gallega», y Juana, «la chinita». Las protagonistas llevan los mismos nombres que las abuelas de la autora, a quienes se les dedica el libro.

En segundo lugar, algunas dedicatorias de esta clase de novelas incluyen algún otro componente en el que se menciona el sacrifico que implicó para el personaje la migración del país de origen (o la dura vida que llevaba allí); personaje que es, a su vez, la persona dedicataria. Retomadas en textos al final del libro, estas dedicatorias establecen una estrecha relación con la instancia prefacial, ya que comparten e, incluso, reiteran información. En este sentido, consideramos prefacio en un modo amplio, como

[...] a toda especie de texto liminar (preliminar o pos-liminar) autoral o alógrafo, que constituye un discurso producido a propósito del texto que sigue o que precede. El «posfacio» será considerado como una variedad de prefacio [...]. La lista de sus parasinónimos [...] de ningún modo exhaustiva: introducción, prólogo, nota, noticia, aviso, presentación, examen, preámbulo, advertencia, preludio, discurso preliminar, exordio, proemio, y para el posfacio: epílogo, postscriptum y otros (Genette, 2001, p. 137).

Los casos más relevantes de esta clase de dedicatorias se encuentran en las novelas de María Teresa Andruetto, Cecilia Pisos y Perla Suez.

Stefano (1997), de Andruetto, es la historia de un adolescente italiano que viaja a la Argentina en una accidentada travesía en barco. Después de unos días en el Hotel de Inmigrantes, próximo al puerto de Buenos Aires, se traslada hacia Santa Rosa, 
La Pampa, donde se suma a la gira de un circo. En medio de situaciones vinculadas con la amistad y con el despertar sexual, el protagonista se entera de la muerte de su madre y vive la confrontación entre el hambre vivido en Europa y las posibilidades de progreso que encuentra en América. La dedicatoria de la novela dice: "A mi padre». Al final del libro, en un texto de una página sin título y con su firma, la autora pone en relación acciones y personajes de la novela con situaciones de la vida de su padre e, incluso, de la suya propia. Sin embargo, recalca que Stefano (1997) carece del carácter biográfico que pareciera dar a entender.

Finalmente, la autora confronta el contexto de la época de la historia con el de la escritura de su novela y de recepción por parte de los/as lectores/as juveniles:

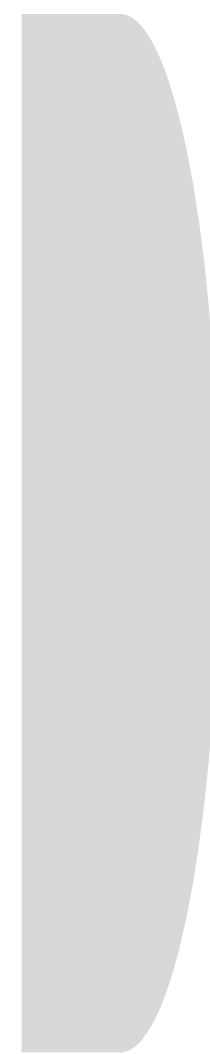

Soy hija de un partisano que llegó desde el norte de Italia a la Argentina, en 1948, y por una sucesión de circunstancias más o menos azarosas, se instaló en un pueblo de la pampa húmeda, donde nací, y donde vivió toda su vida. [...] A poco de venir murió su madre y luego otros y otros, hasta que cada vez se hizo más fuerte la idea de ya no regresar.

Aunque Stefano no relata la vida de mi padre, hay muchas cosas de él en el libro, cosas desperdigadas aquí y allá, sobre todo pequeñas anécdotas y rasgos familiares como [...] el hambre cuyo fantasma acosó a los inmigrantes para siempre, o las comidas que se comían en casa, o las canciones que se cantaban en el puerto, o el nombre de ciertos pueblos por donde sé que él pasó, el título mismo del libro que replica su nombre.

Si un libro es un modo de conocer, una manera de penetrar en el mundo y buscar un sitio que nos corresponde en él, Stefano me permitió recuperar la sensación de hambre, de desarraigo, extrañamiento, de hombres y mujeres que, tal como los que hoy se marchan, ayer llegaban buscando una vida mejor (Andruetto, 1997, p. 91).

Como si no hubiera que cruzar el mar (2005), de Pisos, se presenta como una serie de cartas escritas por Carolina, una niña argentina de doce años que viaja a Madrid a comienzos del siglo XXI, y por María, su bisabuela, que viaja en barco de España hacia la Argentina a mediados del siglo XX. La novela está dedicada «a la memoria de María y Ramón, los verdaderos, mis abuelos. Para Neno». Después de la novela, bajo el subtítulo "De viaje, de libro», Pisos cuenta sobre uno de sus abuelos, 
los verdaderos María y Ramón, y hace hincapié en las instancias de escritura de la novela. De hecho, explicita algunos cambios realizados durante la escritura y destaca cómo es su vínculo con el libro en la actualidad:

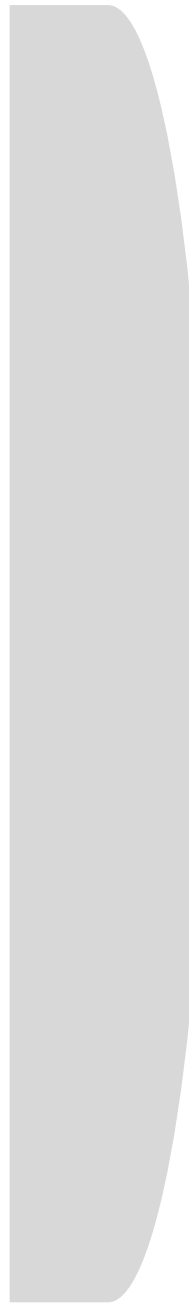

Cuando empecé a escribir Como si no hubiera que cruzar el mar, me ponía un rato en la cabeza de Carolina y un rato, en la de María. Y así, a un lado y otro, se iban armando los capítulos. Les preparaba a las dos situaciones iguales o parecidas, para ver qué salía de cada una, pero las mantenía todo el tiempo separadas. Hasta que un día, creo que a la altura del capítulo "Agujero negro», ellas empezaron a conversar y yo, que había quedado en medio, muda, las escuchaba hablarse como viejas conocidas.

Que en una familia haya idas y venidas, despedidas y reencuentros, parece algo muy común, especialmente en estos días, en este país. Lo que nunca va a ser común es lo que cada uno que parte siente a la hora de irse, de estar yéndose... y de eso trata este libro a dos voces, la de Caro y la de María del Pilar, que se hablan dentro de mi corazón como si para preguntarse y contestarse no tuvieran que cruzar el tiempo, el mar.

Las dos cuentan historias, hacia atrás y hacia adelante, historias de mi familia. Y cada vez que estas historias parten, yo viajo con ellas, con el boleto doble de la esperanza y la tristeza. Ahora que llegaste hasta aquí, lector, tan cerca de mis palabras, te invito a que te prepares también, a que estés atento: nunca se sabe dónde empieza una historia, dónde termina un viaje. Después de todo, un libro y un viaje, no son cosas tan distintas: el riesgo y la maravilla acechan a cada minuto, en cada página (Pisos, 2005, pp. 209-210).

En Memorias de Vladimir (2007 [1992]), de Perla Suez con ilustraciones de María Rojas, su protagonista narra los terribles episodios de su infancia en Rusia que hacen que deba huir hacia la Argentina, su viaje en barco -al que subió con un gallo- y su nueva vida en Entre Ríos. Este libro presenta la siguiente dedicatoria: «A mi abuelo Froique, que anduvo "con los pies sobre la tierra, la frente muy alta, y el cielo sobre la cabeza"*. *Frase de la vieja cultura popular hebrea» (p. 7). Esta mención del abuelo, que se hace en una dedicatoria que recupera la tradición oral, es retomada en las últimas páginas del libro en una sección que lleva como título el nombre de la autora. Allí, a partir de algunos datos autobiográficos, Suez pone en relación su vida con las acciones narradas en la novela: 
Nací en Córdoba y me crié en Basavilbaso, un pueblo de Entre Ríos, cerca de donde transcurre la historia de Vladimir.

Soy nieta de inmigrantes judíos que escaparon de Rusia en la época del zar Nicolás II, a fines del siglo XIX.

Cuando yo tenía doce años, no ignoraba las matanzas que habían ocurrido a la familia de mi abuelo y tampoco desconocía cómo mi abuelo escapó en un barco a América. Sé que la memoria es sabia, y sé que ustedes no desconocen lo que ocurrió y puede ocurrir en cualquier lugar y en cualquier época (Suez, 2007, p. 71).

En ese mismo texto, la autora expone una reflexión sobre el proceso de escritura de la novela en el que remarca que un aspecto de la vida de su padre (la llegada a la Argentina con un gallo como mascota) devino en un elemento que disparó su imaginación. Además, explicita las dificultades que tuvo para reconstruir lo dicho por el personaje, pero enfatiza, a su vez, el carácter ficcional de la novela y hace hincapié en la libertad que eso genera en su rol de autora. Por último, apela al/la lector/a juvenil para apropiarse del relato y destaca que este libro funcionó como un desprendimiento, como un cierre de su propia historia:

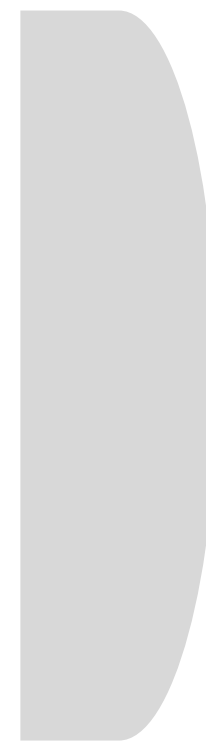

Cuando escribía esta novela imaginé a ese niño que era mi abuelo llegando al puerto de Buenos Aires con un gallo en sus brazos. Es probable que Vladimir hable aquí y diga cosas que no decía mi abuelo; pero en una historia todo es posible.

Cuando escribía Memorias de Vladimir -no me fue fácil encontrar las palabras precisas que dijeran lo que tenían que decir-, mi pretensión era importante. Confío en que ustedes podrán entrar en la vida de Vladimir y disfrutarla sintiéndola como propia.

[...] en cuanto a la realidad de mi infancia, ahora se esfuma. Es sombra de las palabras que ya no están, pero quedan Memorias de Vladimir y Vladimir, que se han desprendido de mí para siempre (Suez, 2007, pp. 71-73).

Las dedicatorias de Andruetto, Pisos y Suez se complementan, se entrecruzan con textos ubicados al final de los libros que se presentan, a su vez, bajo distintas denominaciones o, incluso, ninguna. Pueden relacionarse, por lo tanto, con esas excepciones a las que Genette refiere cuando afirma: «Salvo usurpaciones 
adicionales por sobre las del prefacio, su función propia se agota en esta exposición» (2001, p. 116). Estos serían casos de esa usurpación, ya que ciertos datos de estas dedicatorias dan pie a información ampliada a la que se accede mediante la lectura de los prefacios.

Genette afirma que uno de los rasgos de las dedicatorias que tiende a desaparecer a comienzos del siglo XIX es la «forma desarrollada de la epístola elogiosa» (p. 116), que incluía la posibilidad de contener otros mensajes como «informaciones sobre las fuentes y las génesis de la obra, o comentarios sobre su forma o su significación, por lo cual la función de la dedicatoria avanza claramente sobre la del prefacio» (p. 116). Si bien no puede afirmarse que las novelas juveniles sobre inmigración recuperan la epístola elogiosa, sí podemos sostener que sus dedicatorias establecen una relación con los prefacios que mantienen funciones similares.

En los tres casos, las dedicatorias cumplen, también, la función de anticipar contenidos de los prefacios y estos últimos refuerzan lo mencionado en las dedicatorias, en un juego de lecturas complementarias. Dado que «es el objetivo de la lectura el que [...] define el carácter paratextual o textual de algunos elementos» (Alvarado, 2006, p. 10), en la relación entre dedicatorias y prefacios cada uno de estos elementos funciona como paratexto del otro, según en cuál esté orientada la lectura. En consecuencia, leer esas novelas implica leer, también, sus dedicatorias y sus prefacios. A partir de esto, podemos identificar las operaciones que realizan las autoras: en primer lugar, redactan una dedicatoria afectiva que, en algunos casos, es una dedicatoria al héroe y, al mismo tiempo, motivada. De esta manera, establecen una relación entre dedicatarios y protagonistas de las obras. En segundo lugar, luego de las novelas, en algún tipo de prefacio, mencionan componentes biográficos, pero los matizan explicitando el carácter ficcional de la novela, independiente de los hechos reales. Por último, realizan una apelación al/la lector/a y, a modo de reflexión, concluyen sobre cuál es el vínculo generado con su propia novela.

Lo desarrollado anteriormente es sistematizado en la Figura 1. En la primera columna, se enumeran las operaciones realizadas por las autoras, según el orden de aparición en sus libros, que no necesariamente implica que se hayan realizado en ese orden durante el proceso de escritura. En las columnas siguientes, se citan las dedicatorias completas y fragmentos seleccionados de los prefacios. 


\begin{tabular}{|c|c|c|c|}
\hline Operaciones & $\begin{array}{c}\text { Stefano, } \\
\text { de María Teresa } \\
\text { Andruetto }\end{array}$ & $\begin{array}{l}\text { Como si no hubiera } \\
\text { que cruzar el mar, } \\
\text { de Cecilia Pisos }\end{array}$ & $\begin{array}{c}\text { Memorias de Vladimir, } \\
\text { de Perla Suez }\end{array}$ \\
\hline Dedicatoria & A mi padre & $\begin{array}{l}\text { A la memoria de María } \\
\text { y Ramón, los verdaderos, } \\
\text { mis abuelos. Para Neno. }\end{array}$ & $\begin{array}{l}\text { A mi abuelo Froique, } \\
\text { que anduvo «con los pies } \\
\text { sobre la tierra, la frente } \\
\text { muy alta, y el cielo } \\
\text { sobre la cabeza»*. } \\
\text { *Frase de la vieja cultura } \\
\text { popular hebrea }\end{array}$ \\
\hline $\begin{array}{l}\text { Mención } \\
\text { de algún } \\
\text { componente } \\
\text { biográfico }\end{array}$ & $\begin{array}{l}\text { «Hay muchas } \\
\text { cosas de él en } \\
\text { el libro, cosas } \\
\text { desperdigadas } \\
\text { aquí y allá» }\end{array}$ & $\begin{array}{c}\text { «Las dos cuentan } \\
\text { historias, hacia atrás } \\
\text { y hacia adelante, } \\
\text { historias de mi familia.» }\end{array}$ & $\begin{array}{c}\text { «Cuando escribía } \\
\text { esta novela imaginé } \\
\text { a ese niño que era mi } \\
\text { abuelo llegando al puerto } \\
\text { de Buenos Aires con } \\
\text { un gallo en sus brazos.» }\end{array}$ \\
\hline $\begin{array}{l}\text { Explicitación } \\
\text { del carácter } \\
\text { ficcional } \\
\text { de la novela }\end{array}$ & $\begin{array}{l}\text { «Stefano } \\
\text { no relata la vida } \\
\text { de mi padre» }\end{array}$ & $\begin{array}{l}\text { «[...] se iban armando } \\
\text { los capítulos. } \\
\text { Les preparaba a las dos } \\
\text { situaciones iguales } \\
\text { o parecidas, para ver } \\
\text { qué salía de cada una, } \\
\text { pero las mantenía todo } \\
\text { el tiempo separadas.» }\end{array}$ & $\begin{array}{l}\text { «En una historia } \\
\text { todo es posible.» }\end{array}$ \\
\hline $\begin{array}{l}\text { Apelación } \\
\text { al presente } \\
\text { del lector }\end{array}$ & $\begin{array}{l}\text { «Tal como los que } \\
\text { hoy se marchan, } \\
\text { ayer llegaban } \\
\text { buscando } \\
\text { una vida mejor» }\end{array}$ & $\begin{array}{c}\text { «Ahora que llegaste hasta } \\
\text { aquí, lector, tan cerca } \\
\text { de mis palabras, } \\
\text { te invito a que te } \\
\text { prepares también, } \\
\text { a que estés atento: } \\
\text { nunca se sabe dónde } \\
\text { empieza una historia, } \\
\text { dónde termina un viaje.» }\end{array}$ & $\begin{array}{l}\text { «Confío en que ustedes } \\
\text { podrán entrar en la vida } \\
\text { de Vladimir y disfrutarla } \\
\text { sintiéndola como propia.» }\end{array}$ \\
\hline $\begin{array}{l}\text { Reflexión sobre } \\
\text { el vínculo con } \\
\text { su propia obra, } \\
\text { qué significa } \\
\text { para ellas su libro }\end{array}$ & $\begin{array}{c}\text { «Stefano me } \\
\text { permitió recuperar } \\
\text { la sensación } \\
\text { de hambre, } \\
\text { de desarraigo, } \\
\text { extrañamiento» }\end{array}$ & $\begin{array}{c}\text { «De eso trata este libro } \\
\text { a dos voces, la de Caro } \\
\text { y la de María del Pilar, } \\
\text { que se hablan dentro } \\
\text { de mi corazón como } \\
\text { si para preguntarse } \\
\text { y contestarse no tuvieran } \\
\text { que cruzar el tiempo, } \\
\text { el mar.» }\end{array}$ & $\begin{array}{c}\text { «Es sombra de las palabras } \\
\text { que ya no están, } \\
\text { pero quedan Memorias } \\
\text { de Vladimir y Vladimir, } \\
\text { que se han desprendido } \\
\text { de mí para siempre.» }\end{array}$ \\
\hline
\end{tabular}

Figura 1 | Operaciones discursivas en dedicatorias y en prefacios Fuente: elaboración propia 
Si el abordaje de todo tipo de novelas se enriquece cuando la lectura de la obra se realiza en conjunto con sus dedicatorias y sus prefacios, en el caso de las de temática de la inmigración dicha lectura conjunta se torna prácticamente imprescindible. Allí, se ponen en tensión los sentidos de algunas nociones con las que se abordan las novelas para lectores juveniles, tales como autor, narrador, carácter autobiográfico, paratexto e intertextualidad, solo por mencionar algunos. Como si no alcanzara con escribir la novela, estas autoras refuerzan con elementos paratextuales, entrelazados entre ellos, la memoria reconstruida. Es necesario, sin embargo, hacer una aclaración. De ninguna manera se pretende expresar que estos cruces entre las dedicatorias y la instancia prefacial son exclusivos de las novelas que abordan la temática de la inmigración. De hecho, se observa también en novelas que presentan un tono autobiográfico.

A modo de cierre y como ilustración de la riqueza que poseen las dedicatorias de las novelas juveniles argentinas de temática de la inmigración, presentamos la del libro Chaquetein 1880 o el Frankestein criollo (2004), de Marcelo Suárez del Prado. Esta contiene un fragmento que, incluido humildemente entre paréntesis, podría funcionar como un texto autónomo que homenajea a la comunidad gallega instalada en la Argentina, de la que da cuenta la novela.

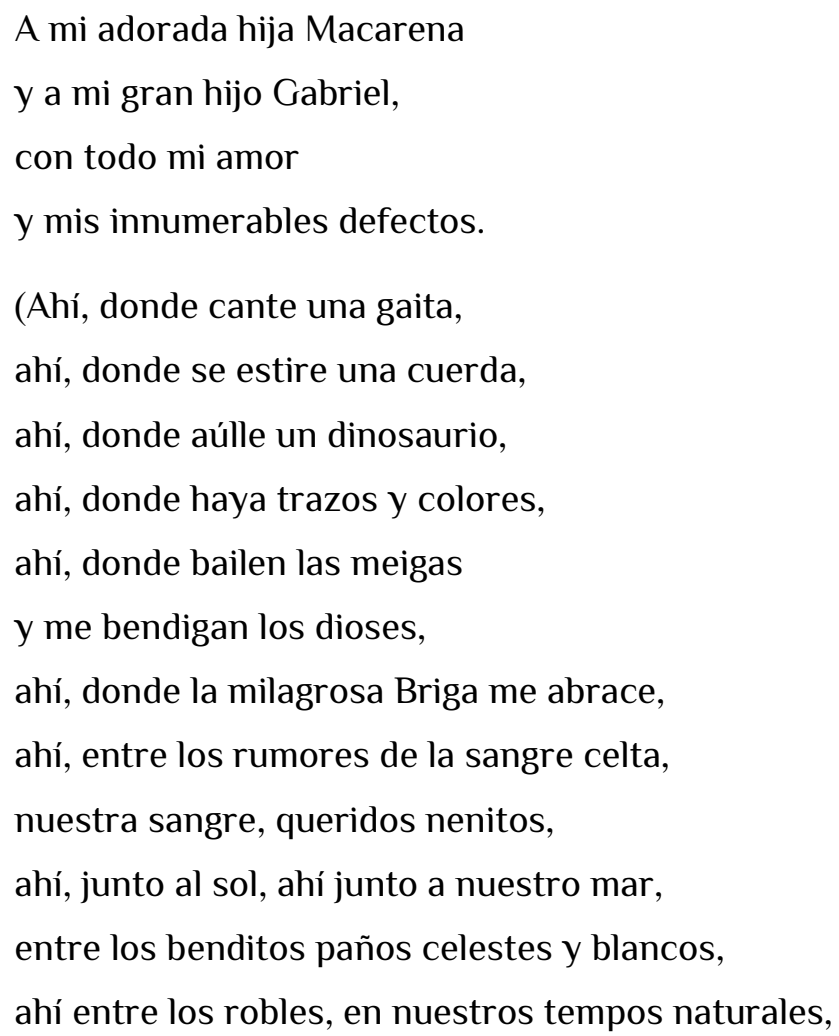


ahí, donde mis enemigos caigan y regresen a la nada,

ahí, donde haya libros y bibliotecas,

ahí, en el incomprensible Universo,

siempre, siempre, miña nena, miño neno, estaremos juntos).

Y a mi prima Cristina, hace tanto tiempo

lo llamó Chaquetein. Y Chaquetein se hizo.

Marcelo Suárez del Prado (2004). Chaquetein 1880 o el Frankestein criollo.

\section{Conclusiones}

Tal como se ha demostrado en las páginas anteriores, este trabajo pretende superar la carencia en el abordaje de las dedicatorias en las novelas juveniles argentinas. Para ello, relevamos las dedicatorias que figuran en aquellas obras publicadas desde 1983 hasta la actualidad. En primer lugar, identificamos una clase de dedicatoria que podríamos denominar afectiva, que se presenta como una de las más habituales en las novelas juveniles. Junto con ella, caracterizamos la dedicatoria colectiva, como aquella en la que se mencionan varias personas. Por otro lado, destacamos una ausencia en las novelas juveniles argentinas: las dedicatorias a lo Castillo, aquel conjunto de dedicatorias que evocaría a la/s misma/s persona/s en todos los libros.

A continuación, destacamos las dedicatorias motivadas, que explicitan una vinculación con el contenido del libro que preceden, en sus dos variantes: con dedicatarios individualizados y con dedicatorios colectivos. Dentro de esta segunda variante, se registran algunas dedicatorias que presentan el libro como una especie de revancha. Esta característica nos permitió hacer hincapié en las dedicatorias comprendidas en la literatura de temas difíciles. Más adelante, observamos las dos modalidades de dedicatorias en las que el/la autor/a menciona a otro/a escritor/a: por un lado, las que indican afinidad o amistad; por otro, las que funcionan como procedimiento para situarse junto con escritores ya canónicos.

Al analizar, en particular, las dedicatorias de las novelas juveniles sobre la inmigración, se hizo hincapié en la riqueza que genera su lectura en conjunto con los otros elementos paratextuales de las obras. La productividad de estas dedicatorias se ejemplificó con énfasis en tres novelas: Stefano (1997), de Andruetto, 
Como si no hubiera que cruzar el mar (2005), de Cecilia Pisos, y Memorias de Vladimir (2007 [1992]), de Perla Suez. Allí se observó que estas dedicatorias establecen una relación estrecha con la instancia prefacial, en efecto, presentan información compartida. Esto permitió identificar que mantienen funciones similares a las de la epístola elogiosa, supuestamente en desuso.

Consideramos que el abordaje de las dedicatorias de las novelas juveniles argentinas resulta un original aporte tanto a los estudios de los paratextos como a los del campo de la literatura infantil y juvenil. En ese sentido, se reconoce que este estudio puede leerse como respuesta a la necesidad planteada por Teresa Colomer (2010): «Es evidente que un análisis más detallado de la novela juvenil en los distintos países puede servir de ejemplo del peso que [...] el contexto sociocultural propio ejerce en cualquier género literario infantil y juvenil» (p. 181).

En este artículo se evoca la instancia del contacto que los/as lectores/as tenemos con un libro que llega a nuestras manos por primera vez. Ese contacto que se establece al sostenerlo, al tocarlo y al olerlo, para abrirlo y para husmear si hay dedicatoria. Cuando la encontramos y la leemos, inferimos, suponemos y reconstruimos posibles historias no explicitadas en ese breve texto. De esta manera, ampliamos sentidos, complementamos relatos y reordenamos biografías. Así, llegamos a darnos cuenta de que así como una dedicatoria se lee en el marco de un libro, leer un libro implica leer también su dedicatoria.

\section{Referencias}

Aliano Casales, S. y Dell'Orto, F. (2018). Leru, leru. Montevideo, Uruguay: Más pimienta.

Alvarado, M. (2006). Paratexto. Ciudad Autónoma de Buenos Aires, Argentina: Eudeba.

Bloom, H. (1994). El canon occidental. Barcelona, España: Anagrama.

Bornemann, E. (1994). Socorro. Ciudad Autónoma de Buenos Aires, Argentina: Alfaguara. 
Castillo, A. (1997). Cuentos completos. Ciudad Autónoma de Buenos Aires, Argentina: Alfaguara.

Colomer, T. (2010). Introducción a la literatura infantil y juvenil. Madrid, España: Síntesis.

Genette, G. (2001). Umbrales. Ciudad Autónoma de Buenos Aires, Argentina: Siglo XXI.

Kunz, M. (2003). La inmigración en la literatura juvenil. En M. Kunz y U. Vences (2003), Europa necesita migrantes. España y Latinoamérica como ejemplos de las migraciones (pp. 38-56). Berlín, Alemania: Tranvia.

Labeur, P. (2019). Dar para leer. El problema de la selección de textos en la enseñanza de la lengua y la literatura. Ciudad Autónoma de Buenos Aires, Argentina: UNIPE- Editorial Universitaria.

Lluch, G. (2004). Cómo analizamos relatos infantiles y juveniles. Bogotá, Colombia: Norma.

Martínez Pueyo, C. (2016). El chico de las estrellas. Ciudad Autónoma de Buenos Aires, Argentina: Destino.

Perriconi, G. (2005). La literatura para chicos y jóvenes y los temas «difíciles». Lectura y Vida. Revista latinoamericana de lectura, 26(2), 6470. Recuperado de http://www.lecturayvida.fahce.unlp.edu.ar/numeros/a26n2/26_02_Perrico ni.pdf

Vulponi, A. (2010). «Hacia una lectura etnográfica: diálogos de "memorias” en Stefano de María Teresa Andruetto». En C. Blake y V. Sardi (Comps.), Literatura argentina e infancia: Un caleidoscopio de poéticas (pp. 261276). Ciudad Autónoma de Buenos Aires, Argentina: Vuelta a casa. 


\section{Fuentes}

Andruetto, M. (1997). Stefano.

Ciudad Autónoma de Buenos Aires, Argentina: Sudamericana.

Andruetto, M. (2016) [2005]. Veladuras.

Ciudad Autónoma de Buenos Aires, Argentina: Norma.

Áverbach, M. (2003). El año de la vaca.

Ciudad Autónoma de Buenos Aires, Argentina: Sudamericana.

Áverbach, M. (2009). Una cuadra.

Ciudad Autónoma de Buenos Aires, Argentina: Adriana Hidalgo.

Barberis, A. (1995). Cruzar la noche.

Ciudad Autónoma de Buenos Aires, Argentina: Colihue.

Barberis, A. (2015). El infierno de los vivos.

Ciudad Autónoma de Buenos Aires, Argentina: Colihue.

Bialet, G. (1997). Los sapos de la memoria.

Córdoba, Argentina: CB ediciones.

Bialet, G. (2004). Si tu signo no es cáncer.

Ciudad Autónoma de Buenos Aires, Argentina: Norma.

Birmajer, M. (1992). Un crimen secundario.

Ciudad Autónoma de Buenos Aires, Argentina: Colihue.

Birmajer, M. (1993). Derrotado por un muerto.

Ciudad Autónoma de Buenos Aires, Argentina: Colihue.

Bodoc, L. (2000). Los días del venado.

Ciudad Autónoma de Buenos Aires, Argentina: Norma.

Bodoc, L. (2013). El espejo africano.

Ciudad Autónoma de Buenos Aires, Argentina: SM.

Bodoc, L. (2013). El perro del peregrino.

Ciudad Autónoma de Buenos Aires, Argentina: Alfaguara.

Bombara, P. (2005) [2002]. El mar y la serpiente.

Ciudad Autónoma de Buenos Aires, Argentina: Norma. 
Bombara, P. (2015). La chica pájaro.

Ciudad Autónoma de Buenos Aires, Argentina: Norma.

Bombara, P. (2016). Lo que guarda un caracol.

Ciudad Autónoma de Buenos Aires, Argentina: Santillana.

Borges, J. (1989) [1952]. Kafka y sus precursores. En Obras completas.

Vol. II. Ciudad Autónoma de Buenos Aires, Argentina: Emecé.

Boselli, A. y Bellina (2015). La princesa guerrera.

Ciudad Autónoma de Buenos Aires, Argentina: Muchas Nueces.

Carreras de Sosa, L. (2007). El juramento de los Centenera.

Ciudad Autónoma de Buenos Aires, Argentina: Edelvives.

Cinetto, L. (2019). Mientras no muera tu nombre.

Ciudad Autónoma de Buenos Aires, Argentina: Norma.

De Santis, P. (2003). El inventor de juegos.

Ciudad Autónoma de Buenos Aires, Argentina: Alfaguara.

De Santis, P. (2008). El buscador de finales.

Ciudad Autónoma de Buenos Aires, Argentina: Alfaguara.

Dedé, M. (2015). El comedor de las tinieblas.

Ciudad Autónoma de Buenos Aires, Argentina: Estrada.

Dellutri, E. (2018). Koi.

Ciudad Autónoma de Buenos Aires, Argentina: Norma.

Ferrari, A. (2003). El complot de las flores.

Ciudad Autónoma de Buenos Aires, Argentina: SM.

Ferrari, A. (2004). Café solo.

Ciudad Autónoma de Buenos Aires, Argentina: SM.

Ferrari, A. (2014) [2007]. El camino de Sherlock.

Ciudad Autónoma de Buenos Aires, Argentina: Alfaguara.

Ferrari, A. (2015) [2004]. La rebelión de las palabras.

Ciudad Autónoma de Buenos Aires, Argentina: Santillana.

Giardinelli, M. (2006) (Comp.). Cuentos para seguir creciendo para los estudiantes que terminan la Educación Secundaria. Ciudad Autónoma de Buenos Aires, Argentina: Eudeba. 
González, E. y Aguirre, O. (2007). Graffiti ninja.

Ciudad Autónoma de Buenos Aires, Argentina: Siete Vacas.

Huidobro, N. (2004). Octubre, un crimen.

Ciudad Autónoma de Buenos Aires, Argentina: SM.

Huidobro, N. (2010). El pan de la serpiente. Lazos de amistad durante la inmigración. Ciudad Autónoma de Buenos Aires, Argentina: Norma.

Kolesnicov, P. (2017). Me enamoré de una vegetariana.

Ciudad Autónoma de Buenos Aires, Argentina: Norma.

Maine, M. (2005). El hijo de la libertad.

Ciudad Autónoma de Buenos Aires, Argentina: Norma.

Mamone, J. I. e Ibarra, M. (2018). Yael y la casa violeta.

Ciudad Autónoma de Buenos Aires, Argentina: Julia Inés Mamone.

Maritano, A. (1984). El visitante.

Ciudad Autónoma de Buenos Aires, Argentina: Colihue.

Maritano, A. (1986). Vaqueros y trenzas.

Ciudad Autónoma de Buenos Aires, Argentina: Colihue.

Maritano, A. (1997). Pretextos para un crimen.

Ciudad Autónoma de Buenos Aires, Argentina: Colihue.

Montes, G. (1994). Otroso.

Ciudad Autónoma de Buenos Aires, Argentina: Alfaguara.

Muslip, E. (1997). Hojas de la noche.

Ciudad Autónoma de Buenos Aires, Argentina: Colihue.

Orgambide, J. (1998). Las botas de Anselmo Soria.

Ciudad Autónoma de Buenos Aires, Argentina: Ángel Estrada y Cía.

Piñeiro, C. (2010). El fantasma de las invasiones inglesas.

Ciudad Autónoma de Buenos Aires, Argentina: Norma.

Pisos, C. (2005). Como si no hubiera que cruzar el mar. Ciudad Autónoma de Buenos Aires, Argentina: Alfaguara.

Potes, M. (2018). El campo deportivo.

Ciudad Autónoma de Buenos Aires, Argentina: SM. 
Ramos, P. (2015). El sueño de los murciélagos.

Ciudad Autónoma de Buenos Aires, Argentina: Alfaguara.

Rodó, J. (1993). Ariel y Proteo selecto.

Caracas, Venezuela: Biblioteca Ayacucho.

Saint-Exupéry, A. (1987) [1943]. El principito.

Ciudad Autónoma de Buenos Aires, Argentina: Emecé.

Santa Ana, A. (1998). Los ojos del perro siberiano.

Ciudad Autónoma de Buenos Aires, Argentina: Norma.

Schillat, M. (2013). Cartas a Messi.

Ushuaia, Argentina: Paraviento.

Schujer, S. (1991). Palabras para jugar.

Ciudad Autónoma de Buenos Aires, Argentina: Sudamericana.

Shock, S. (2007). Revuelo sur.

Ciudad Autónoma de Buenos Aires, Argentina: Nuevos tiempos.

Shock, S. (2011). Poemario Trans Pirado.

Ciudad Autónoma de Buenos Aires, Argentina: Nuevos tiempos.

Shock, S. (2016). Crianzas.

Ciudad Autónoma de Buenos Aires, Argentina: Muchas nueces.

Solá, J. (2019). Naranjo en flúo.

Ciudad Autónoma de Buenos Aires, Argentina: Sudestada.

Sosa Villada, C. (2015). La novia de Sandro.

Córdoba, Argentina: Caballo negro.

Sosa Villada, Camila (2018). El viaje inútil. Trans/escritura.

Córdoba, Argentina: DocumentA/Escénicas.

Sosa Villada, C. (2019). Las malas.

Ciudad Autónoma de Buenos Aires, Argentina: Tusquets.

Suárez del Prado, M. (2004). Chaquetein 1880 o el Frankestein criollo. Ciudad Autónoma de Buenos Aires, Argentina: Alfaguara.

Suez, P. (2007) [1992]. Memorias de Vladimir.

Ciudad Autónoma de Buenos Aires, Argentina: Alfaguara. 
Sukaczer, V. (2015). Los nombres prestados.

Ciudad Autónoma de Buenos Aires, Argentina: Nube de tinta.

Unrein, C. (2019). Pendeja. Diario de una adolescente trans.

Ciudad Autónoma de Buenos Aires, Argentina: Chirimbote.

Unrein, C. (2020). Fatal. Una crónica trans.

Ciudad Autónoma de Buenos Aires, Argentina: Planeta.

Vaccarini, F. (2015). Cazadores.

Ciudad Autónoma de Buenos Aires, Argentina: Edelvives.

Vasconcelos, J. de (2001) [1968]. Mi planta de naranja lima.

Ciudad Autónoma de Buenos Aires, Argentina: El Ateneo.

\section{Notas}

1 Si bien no se trata de una novela, sino de una antología de cuentos, resulta pertinente mencionar el caso de Cuentos para seguir creciendo para los estudiantes que terminan la Educación Secundaria (2006), compilados por Mempo Giardinelli. El Ministerio de Educación de la Nación repartió ejemplares de este libro a los/as alumnos/as, cuya dedicatoria los/as mencionaba. Esta dedicatoria de ejemplar (pero impresa), que no estaba firmada, presentó distintas versiones.

2 En cuanto a la dedicatoria que figura al final del libro, después del texto literario, Genette (2001) afirma que es «infinitamente más rara, pero tiene sus cartas de nobleza» (p. 109) y menciona algunos casos. En el ámbito de la literatura infantil, quizás el ejemplo más reciente sea el libro uruguayo Leru leru (2018), de Susana Aliano Casales con ilustraciones por Francesca Dell'Orto. Esta obra, que aborda la problemática del bullying en la escuela primaria, finaliza con una inquietante dedicatoria: «A Edgar, a quien seguramente hice daño en aquel juego de niños».

3 En cada categoría, las dedicatorias aparecen por orden alfabético según apellido de autor/a.

4 Asimismo, resulta pertinente indicar una particularidad que presenta una edición juvenil de los cuentos de Abelardo Castillo. Se trata de la antología El candelabro de plata y otros cuentos (2006) que, con prólogo de Guillermo Saccomano y estudio de Aníbal Jarkowski, la editorial Alfaguara incluyó en su serie roja. En esta publicación se omite la habitual dedicatoria que Castillo le hacía a su esposa, la escritora Sylvia Iparraguirre. Nos encontraríamos aquí con un caso de «supresión ulterior» (Genette, 2001, p. 110). Esta situación resulta interesante porque nos lleva a preguntarnos cómo operan las ediciones juveniles en lo referente a lo paratextual.

5 Esta dedicatoria presenta una particularidad: también figura en la tapa del libro. Además, en la página siguiente, antes del comienzo de la obra, figura la siguiente nota: «Estas no son las botas del gato con botas, sino las botas de Anselmo Soria, el abuelo de mi abuelo. Las encontré en el altillo. Ahora son mías, como la historia que les cuento». Sobre la lectura de materiales que presentan estas 
características, Genette (2001) afirma que «se puede también dedicar muy simplemente al lector, y sin duda ciertos avisos al lector deberían ser leídos como epístolas dedicatorias tanto como prefacios» (p. 114).

6 La literatura infantil y juvenil de temática LGBTIQ presenta dos interesantes ejemplares de este tipo de dedicatorias: por un lado, la de El chico de las estrellas (2016), de Martínez Pueyo, expuesta al comienzo de este trabajo; por otro, la del libro infantil argentino La princesa guerrera (2015), de Amalia Boselli y Ballina: «A todxs nosotrxs, lxs niñxs que fuimos, que quisimos leer estas historias... y hoy las escribimos. Amalia Boselli».

7 En las páginas de los agradecimientos, la autora expresa: «Quizás porque en esta novela se entrelazan la ficción con una parte importante de mi vida, no puedo dejar de agradecer a los que me acompañaron en el proceso de soñarla, escribirla y hacerla realidad. En primer lugar a mis hijos Sol, Juani y Flor, porque me hicieron comprender que necesitaba conocer la historia de mi hermana Patricia y me ayudaron a derrumbar el muro de dolor para poder contarla. [...] A mis padres porque nos une la pena de no tener a Patricia y porque me prestaron la foto de ella para la portada [...]" (Cinetto, 2019, pp. 181-182).

8 En la literatura infantil, Silvia Schujer (1991) realiza un procedimiento similar en la dedicatoria a Oliverio Girondo en su libro Palabras para jugar. 